\title{
Efecto de los aditivos en la formación y estabilidad térmica a baja temperatura del titanato de aluminio
}

\author{
I. BARRIOS DE ARENAS ${ }^{1}$, F. ARENAS' ${ }^{1}$, S.-A. CHO ${ }^{2}$, S. MARTÍNEZ ${ }^{3}$, R. SICARDI' 1. \\ ${ }^{1}$ Departamento de Tecnología de Materiales, I.U.T. "Dr. Federico Rivero Palacio", Apartado Postal 40347. \\ Caracas 1040-A, Venezuela. \\ ${ }^{2}$ Instituto Venezolano de Investigaciones Científicas, Apartado Postal 21827. Caracas 1020A, Venezuela. \\ ${ }^{3}$ Departamento de Ciencia de los Materiales, Universidad Simón Bolívar, Apartado 89000, Caracas 1080. Venezuela
}

\begin{abstract}
En este trabajo se presenta el estudio del efecto de la adición de $3 \%$ en peso de $\mathrm{V}_{2} \mathrm{O}_{5}, \mathrm{Na}_{2} \mathrm{O}, \mathrm{CaO}$ y FeTiO 3 en la estabilización térmica del Titanato de Aluminio $\left(\mathrm{Al}_{2} \mathrm{TiO}_{5}\right)$. El procesamiento de las muestras fue mediante la molienda en seco de los polvos de alúmina y titania, mezcla vía húmeda con alcohol isopropílico y sinterización a $1400^{\circ} \mathrm{C}$ de los compactos prensados uniaxialmente a $300 \mathrm{MPa}$. Una vez sinterizadas las muestras fueron tratadas térmicamente a $1000^{\circ} \mathrm{C}$ durante 12 y 20 horas para evaluar la descomposición del $\mathrm{Al}_{2} \mathrm{TiO}_{5}$. La microestructura de las probetas se analizó mediante Microscopía Electrónica de Barrido (MEB) y las fases presentes se determinaron mediante Difracción de Rayos X (DRX) y Espectroscopía de Rayos X por dispersión de Energía (EDX). Esta caracterización evidenció el efecto beneficioso de la adición de $\mathrm{FeTiO}_{3}(\mathrm{Ilmenita)}$ en la formación y estabilización térmica del $\mathrm{Al}_{2} \mathrm{TiO}_{5}$.
\end{abstract}

\section{Palabras clave: titanato de aluminio,sinterización reactiva, estabilización térmica}

\section{Additives effect on the formation and thermal stability of aluminum titanate at low temperature}

This work presents the study of the effect of $3 \mathrm{wt} \% \mathrm{~V}_{2} \mathrm{O}_{5}, \mathrm{Na}_{2} \mathrm{O}, \mathrm{CaO}$ and $\mathrm{FeTiO}_{3}$ on the stabilization of Aluminum Titanate $\left(\mathrm{Al}_{2} \mathrm{TiO}_{5}\right)$. Samples were prepared by the conventional processing route of dry ball milling followed by wet mixing and final uniaxial pressing at $300 \mathrm{MPa}$ before sintering at $1400^{\circ} \mathrm{C}$. Specimens were heat treated at $1000^{\circ} \mathrm{C}$ during 12 and 20 hours in order to evaluate $\mathrm{Al}_{2} \mathrm{TiO}_{5}$ decomposition. The microstructure was observed by Scanning Electron Microscopy (SEM) and phases present were determined by X Ray Diffraction (XRD) and Energy Dispersive Spectroscopy (EDS). It has been elucidated the effect of $\mathrm{FeTiO}_{3}$ (Ilmenite) on the improvement of the formation and thermal stabilization of $\mathrm{Al}_{2} \mathrm{TiO}_{5}$.

Keywords: aluminum titanate, reactive sintering, thermal stabilization

\section{INTRODUCCIÓN}

El Titanato de Aluminio $\left(\mathrm{Al}_{2} \mathrm{TiO}_{5}\right)$ se ha convertido en un material cerámico de gran importancia a nivel ingenieril debido a su bajo coeficiente de expansión térmica $\left(0,8-1,5 \times 10^{-6}\right.$ $\left.{ }^{\circ} \mathrm{C}^{-1}\right)$, alta resistencia al choque térmico $(500 \mathrm{~W} / \mathrm{m})$, baja conductividad térmica $(1,5 \mathrm{~W} / \mathrm{mK})$ y su alto punto de fusión $\left(1850^{\circ} \mathrm{C}\right)$, otorgándole un gran potencial en aplicaciones estructurales. Este material es producido por la sinterización reactiva de la mezcla equimolar de polvos de alúmina $\left(\mathrm{Al}_{2} \mathrm{O}_{3}\right)$ y titania $\left(\mathrm{TiO}_{2}\right)$ por encima de $1300^{\circ} \mathrm{C}$. $\mathrm{El} \mathrm{Al}_{2} \mathrm{TiO}_{5}$ presenta una transformación de fase eutectoide alrededor de $1280^{\circ} \mathrm{C}[1]$. La formación de microgrietas en materiales monofásicos de titanato de aluminio se atribuye a la fuerte anisotropía en su expansión térmica, $\alpha_{a}=-2.9 ; \quad \alpha_{b}=10.3 ; \alpha_{c}=20.1 \times 10^{-6}{ }^{\circ} C^{-1}$, que hace que se generen tensiones térmicas durante el enfriamiento que dan lugar a las mencionadas microgrietas. Las diferencias entre los coeficientes de dilatación de los diferentes ejes de un monocristal de titanato son mayores que entre la pequeña fracción de alumina sin reaccionar y el propio titanato. La aplicación exitosa del material ha dependido de la habilidad para controlar a esta transformación y para enten- der el mecanismo de la descomposición. El estudio de la "estabilización térmica" del titanato de aluminio, sin alterar notablemente sus propiedades, ha sido el objetivo primordial de muchos investigadores[2-12]. Trabajos previos han demostrado el efecto estabilizador de algunos aditivos como $\mathrm{Na}_{2} \mathrm{O}$, $\mathrm{CaO}, \mathrm{MgO}, \mathrm{SiO}_{2}$ y $\mathrm{Fe}_{2} \mathrm{O}_{3}[2,3,5,13,21]$. El término "estabilización térmica" se explica en función de la entrada de cationes como $\mathrm{Mg}$, Fe en la red cristalina de la pseudobrookita. El eutectoide de estas soluciones sólidas está situado a temperaturas menores al del titanato puro, con lo que el margen de estabilidad de las soluciones sólidas aumenta. La síntesis del titanato de aluminio mediante la sinterización reactiva de la mezcla equimolar de los polvos submicrométricos de $\mathrm{Al}_{2} \mathrm{O}_{3} \mathrm{y}$ $\mathrm{TiO}_{2}$ ha demostrado ser efectiva, y aún más efectiva a través de la formación de una solución sólida entre el titanato de aluminio y ciertos aditivos. Por esta razón, este trabajo pretende continuar y enriquecer estos estudios mediante el análisis de la influencia de la adición de $3 \%$ en peso de los aditivos $\mathrm{CaO}, \mathrm{Na}_{2} \mathrm{O}, \mathrm{V}_{2} \mathrm{O}_{5}$ y $\mathrm{Fe}_{2} \mathrm{TiO}_{3}$ en la microestructura y descomposición térmica de $\mathrm{Al}_{2} \mathrm{TiO}_{5}$ 


\section{PARTE EXPERIMENTAL}

TABLA I. MATERIAS PRIMAS UTILIZADAS: ORIGEN Y TAMAÑO DE PARTíCULA PROMEDIO $\left(\mathrm{D}_{50}\right)$

\begin{tabular}{|c|c|c|}
\hline Materia Prima & Proveedor & $\mathbf{D}_{\mathbf{5 0} \%}$ \\
\hline$\alpha-\mathrm{Al}_{2} \mathrm{O}_{3}$ & Alcoa (A-16) & $0.5 \mu \mathrm{m}$ \\
$\mathrm{TiO}_{2}$ (rutilo) & JT-Baker & $0.4 \mu \mathrm{m}$ \\
$\mathrm{FeTiO}_{3}$ & Aguamena, Pto. & $0.6 \mu \mathrm{m}$ \\
$\mathrm{V}_{2} \mathrm{O}_{5}$ & Ayacucho & $0.8 \mu \mathrm{m}$ \\
$\mathrm{Na}_{2} \mathrm{O}$ & ROC-RIC & $0.6 \mu \mathrm{m}$ \\
$\mathrm{CaO}$ & ROC-RIC & $0.6 \mu \mathrm{m}$ \\
\hline
\end{tabular}

La mezcla equimolar de los polvos submicrométricos de $\mathrm{Al}_{2} \mathrm{O}_{3}$ y $\mathrm{TiO}_{2}$ fue realizada mediante molienda en húmedo durante 5 horas con alcohol isopropílico en un molino de bolas de alúmina. La suspensión proveniente del molino fue secada en una estufa a $100^{\circ} \mathrm{C}$ durante 24 horas. Luego, los aditivos fueron incorporados en un $3 \%$ en exceso a las cantidades pesadas de la mezcla equimolar de $\mathrm{Al}_{2} \mathrm{O}_{3}$ y $\mathrm{TiO}_{2}$ en un mortero de alúmina. Los polvos con y sin aditivos fueron prensados uniaxialmente a $300 \mathrm{MPa}$, finalmente, las probetas obtenidas (15 $\mathrm{mm}$ de diámetro y $10 \mathrm{~mm}$ de altura, aproximadamente) fueron sinterizadas a una meseta de $1400^{\circ} \mathrm{C}$ durante 3 horas con una velocidad de calentamiento de $5^{\circ} \mathrm{C} /$ min y una velocidad de enfriamiento de $15^{\circ} \mathrm{C} / \mathrm{min}$. La notación para las probetas utilizada en este trabajo se define en la Tabla II.

\begin{tabular}{|c|c|}
\hline Notación de las Muestras & Materiales Iniciales \\
\hline TA & $\mathrm{Al}_{2} \mathrm{O}_{3}+\mathrm{TiO}_{2}$ \\
TAV & $\mathrm{Al}_{2} \mathrm{O}_{3}+\mathrm{TiO}_{2}+\mathrm{V}_{2} \mathrm{O}_{5}$ \\
TAN & $\mathrm{Al}_{2} \mathrm{O}_{3}+\mathrm{TiO}_{2}+\mathrm{Na}_{2} \mathrm{O}$ \\
TAC & $\mathrm{Al}_{2} \mathrm{O}_{3}+\mathrm{TiO}_{2}+\mathrm{CaO}$ \\
TAF & $\mathrm{Al}_{2} \mathrm{O}_{3}+\mathrm{TiO}_{2}+\mathrm{FeTiO}$ \\
\hline
\end{tabular}

TABLA II. NOTACIÓN DE LAS MUESTRAS ELABORADAS.

La densidad aparente y la porosidad de las probetas sinterizadas fue determinada mediante el método de Arquímedes (norma ASTM C-373). Los resultados se presentan en otra publicación [14]. La microestructura de las muestras se analizó mediante microscopía electrónica de barrido (MEB) y las fases presentes se determinaron mediante difracción de rayos $\mathrm{X}$ (DRX) y espectroscopía de rayos X por dispersión de energía (EDX). La difracción de rayos $X$ se realizó bajo las siguientes condiciones de operación: radiación de $\mathrm{CuK} \alpha$, filtro de $\mathrm{Ni}$, velocidad de barrido de $2^{\circ} 2 \theta$ / s y rango de barrido de 20 a $70^{\circ}$.

Las probetas fueron sometidas a tratamientos térmicos durante 12 y 20 horas a una temperatura de $1000^{\circ} \mathrm{C}$ con el objetivo de estudiar la estabilidad térmica de la estructura mediante el cálculo del porcentaje de la fase $\mathrm{Al}_{2} \mathrm{TiO}_{5}$ presente en cada composición antes y después del tratamiento térmico, según los métodos utilizados por Ishitzuka[2] y Pena[15], los cuales establecen una relación entre las intensidades de los principales picos del titanato de aluminio y la titania.

\section{RESULTADOS Y DISCUSIÓN}

La fig. 1 muestra los patrones de DRX para la composición TA antes y después del tratamiento térmico de $12 \mathrm{~h}$. En esta figura se observan los principales picos para las fases $\mathrm{Al}_{2} \mathrm{TiO}_{5}$,
$\mathrm{Al}_{2} \mathrm{O}_{3}$ y $\mathrm{TiO}_{2}$ y la Tabla III presenta el porcentaje calculado correspondiente para cada fase. La descomposición del $\mathrm{Al}_{2} \mathrm{TiO}_{5}$ prevista luego del tratamiento térmico es evidente en la fig. 1 b). La desaparición de los picos principales de la fase $\mathrm{Al}_{2} \mathrm{TiO}_{5}$ en el patrón de difracción de rayos $\mathrm{X}$ y los porcentajes calculados ratifican la descomposición térmica del titanato de aluminio por debajo de $1280^{\circ} \mathrm{C}[1]$. Esta descomposición es ilustrada en las figs. 2 y 3 , en las cuales se observa la microestructura del titanato de aluminio antes y después del tratamiento térmico, respectivamente.

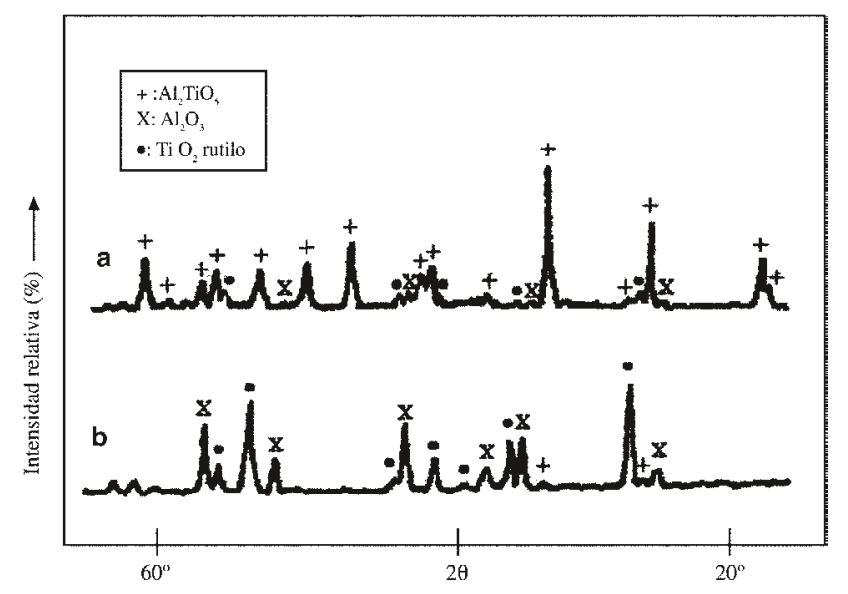

Fig. 1. Patrones de difracción de rayos $\mathrm{X}$ para la composición TA a) antes y b) después del tratamiento térmico.

TABla III. Porcentajes de las fases $\mathrm{Al}_{2} \mathrm{TiO}_{5}, \mathrm{Al}_{2} \mathrm{O}_{3}$ Y TiO 2 PRESENTES EN LA COMPOSICIÓN TA ANTES Y DESPUÉS DEL TRATAMIENTO TÉRMICO DE $12 \mathrm{H} \mathrm{A} 1000^{\circ} \mathrm{C}$

\begin{tabular}{|l|c|c|c|c|}
\hline Composición TA & \multicolumn{2}{|c|}{$\begin{array}{c}\text { Antes del } \\
\text { Tratamiento } \\
\text { Térmico }\end{array}$} & \multicolumn{2}{c|}{$\begin{array}{c}\text { Después del } \\
\text { Tratamiento } \\
\text { Térmico }\end{array}$} \\
\hline$\% \mathrm{Fase} \mathrm{Al}_{2} \mathrm{TiO}_{5}$ & $92.4^{\star}$ & $94.0^{\bullet}$ & $7.2^{\star}$ & $9.1^{\bullet}$ \\
\hline$\% \mathrm{Fase} \mathrm{Al}_{2} \mathrm{O}_{3}$ & $3.8^{\star}$ & $3.0^{\bullet}$ & $45.4^{\bullet}$ & $45.5^{\bullet}$ \\
\hline$\% \mathrm{Fase} \mathrm{TiO}_{2}$ & $3.8^{\star}$ & $3.0^{\bullet}$ & $45.4^{\bullet}$ & $45.5^{\bullet}$ \\
\hline
\end{tabular}

Valor calculado por métodos $\left[2^{\star}, 14^{*}\right]$.

La microestructura típica del titanato de aluminio es mostrada en la fig. 2. Esta está caracterizada por microgrietas, una matriz gris claro, unas zonas pequeñas de color gris oscuro y otras de color blanco que corresponden a titanato de aluminio, alúmina y titania, respectivamente[16], como se pudo verificar con los análisis puntuales de EDX en cada una de estas zonas. La formación de las microgrietas que se observa como característica dominante en la microestructura se debe a la contracción térmica anisotrópica que ocurre durante el enfriamiento luego de la formación de la fase $\mathrm{Al}_{2} \mathrm{TiO}_{5}$. La alúmina y la titania presente en la microestructura pueden ser producto de la descomposición térmica del titanato de aluminio durante el enfriamiento o simplemente residuos de óxidos iniciales que no lograron reaccionar. 


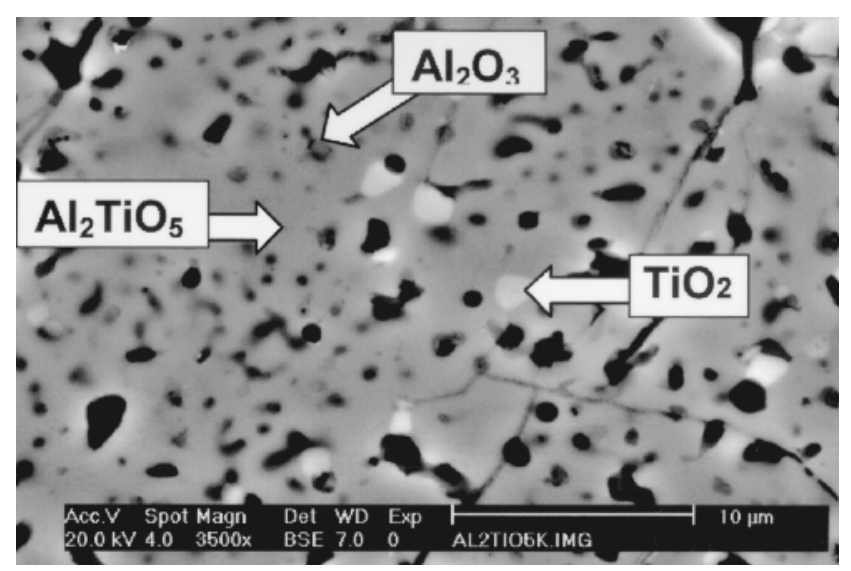

Fig. 2. Microestructura típica del titanato de aluminio. Se distinguen las microgrietas y las zonas de color blanco y gris oscuro embebidas en una matriz de color gris claro.

Las partículas de alúmina y titania presentes en la microestructura del titanato de aluminio son sitios preferenciales que, a temperaturas entre 800 y $1300^{\circ} \mathrm{C}$, favorecen el proceso de nucleación y crecimiento que controlan la cinética de descomposición del titanato de aluminio[17]. Luego del tratamiento térmico de 12 h, la composición TA presenta la microestructura típica de la descomposición del titanato de aluminio (ver fig. 3).

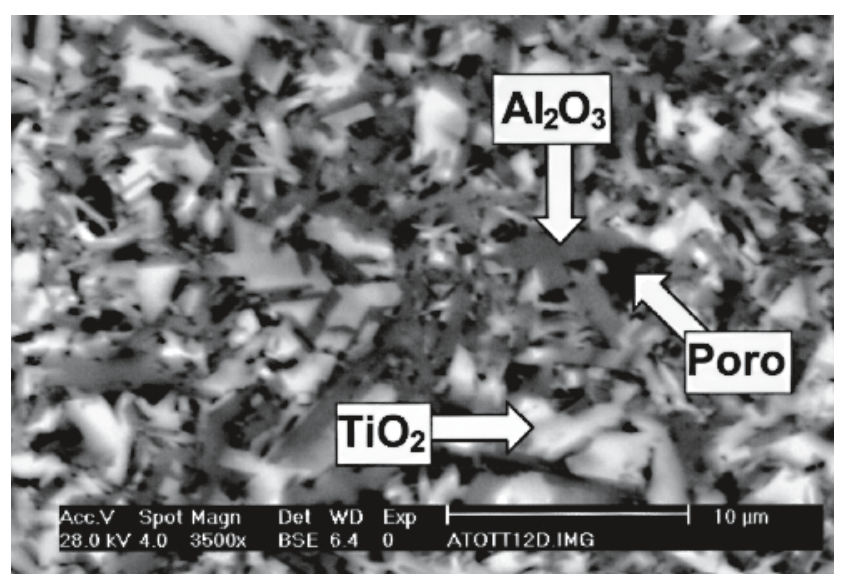

Fig. 3. Microestructura de la composición TA después del tratamiento térmico de $12 \mathrm{~h}$. Obsérvese los granos alargados de color gris oscuro en una matriz de color blanco. También se puede observar la porosidad entre los granos.

Esta descomposición del tipo eutectoide, según Pena y colaboradores[15], involucra una nucleación de las nuevas fases $\left(\mathrm{Al}_{2} \mathrm{O}_{3}\right.$ y $\left.\mathrm{TiO}_{2}\right)$ en los bordes de grano de $\mathrm{Al}_{2} \mathrm{TiO}_{5}$. La microestructura de la descomposición del titanato de aluminio en sus dos compuestos iniciales se caracteriza por partículas de alúmina alargadas y de forma rectangular que crecen dentro de una matriz de titania, como se observa en la fig. 3. Nótese que las partículas exhiben un crecimiento preferencial dentro de una matriz porosa. La porosidad es, predominantemente, intergranular debido a que el crecimiento preferencial de los granos de alúmina y titania durante la descomposición impide la densificación de la probeta de composición TA. Estas características son propias de un proceso de descomposición regido por nucleación y crecimiento, en el cual la nucleación juega un papel más importante que el crecimiento[15,18].
Tabla IV. Porcentaje de la fase $\mathrm{Al}_{2} \mathrm{TiO}_{5}$ PRESEnTE EN las MUestras TAV, TAN, TAC Y TAF ANTES Y DESPUÉS DEL TRATAMIENTO TÉRMICO DE $12 \mathrm{H} \mathrm{A} 1000^{\circ} \mathrm{C}$.

\begin{tabular}{|c|c|c|}
\hline \multirow{2}{*}{ Composición } & \multicolumn{2}{|c|}{$\%$ fase $\mathrm{Al}_{2} \mathrm{TiO}_{5}$ presente } \\
\cline { 2 - 3 } & $\begin{array}{c}\text { Antes del } \\
\text { tratamiento } \\
\text { térmico }\end{array}$ & $\begin{array}{c}\text { Después del } \\
\text { tratamiento } \\
\text { térmico }\end{array}$ \\
\hline $\mathrm{TAV}$ & $85.4^{*}$ & $3.7^{*}$ \\
$\mathrm{TAN}$ & $80.5^{*}$ & $5.3^{*}$ \\
$\mathrm{TAC}$ & $95.3^{*}$ & $18.9^{*}$ \\
$\mathrm{TAF}$ & $100.0^{*}$ & $89.0^{*}$ \\
$\mathrm{TAF}^{+}$ & $100.0^{*}$ & $83.2^{*}$ \\
\hline
\end{tabular}

* Valor promedio entre ambos métodos[2,14].

+ Valor obtenido luego de un tratamiento térmico de $20 \mathrm{~h}$ a $1000^{\circ} \mathrm{C}$.

Los valores reportados en la Tabla IV muestran el efecto de los aditivos en la estabilización del titanato de aluminio y se puede establecer que el aditivo con mayor efecto positivo, bajo las condiciones experimentales estudiadas, es el $\mathrm{FeTiO}_{3}$ (ilmenita). Por lo tanto, el estudio a nivel microestructural del efecto de los aditivos para las diferentes composiciones se realiza en forma comparativa y más extensivamente para la composición TAF.

\subsection{Estudio microestructural y de densificación para las composiciones TAN, TAV, TAC y TAF.}

En la fig. 4 se presenta una vista general de la microestructura de la composición TAV antes del tratamiento térmico. En esta figura se puede apreciar una microestructura similar a la de composición TA con un leve incremento de partículas de los óxidos iniciales y poros. El $85.4 \%$ de fase titanato de aluminio obtenido evidencia que el $\mathrm{V}_{2} \mathrm{O}_{5}$ retarda su formación. Según el diagrama de equilibrio [20] el vanadio no entra en solución sólida en la red del titanato de aluminio sino que reacciona con este descomponiéndolo según la reacción: $\mathrm{Al}_{2} \mathrm{TiO}_{5}+\mathrm{V}_{2} \mathrm{O}_{5} \rightarrow 2 \mathrm{AlVO}_{4}+\mathrm{TiO}_{2}$ formando una fase líquida a temperaturas superiores a $700^{\circ} \mathrm{C}$. La fase líquida disminuye la difusión que rige la eliminación de $\mathrm{Al}_{2} \mathrm{O}_{3}$ y $\mathrm{TiO}_{2}$ residual durante la segunda etapa de la cinética de la formación de $\mathrm{Al}_{2} \mathrm{TiO}_{5}$ descrita por Freudenberg y Mocellin[19].

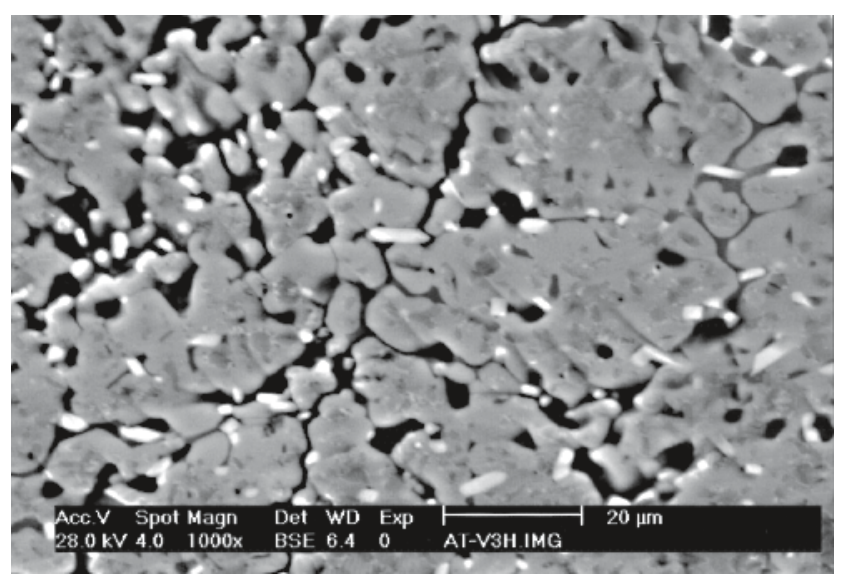

Fig. 4. Microestructura de la composición TAV antes del tratamiento térmico. 
El 3.7\% de fase de $\mathrm{Al}_{2} \mathrm{TiO}_{5}$ presente en la composición TAV después del tratamiento térmico indica que el $\mathrm{V}_{2} \mathrm{O}_{5}$ en exceso en la mezcla inicial de polvos no tuvo efecto beneficioso en la estabilización térmica del titanato de aluminio.

Una microestructura interesante es la mostrada en la fig. 5 perteneciente a la composición TAN. Se pueden observar diversidad de tamaños de granos bien delineados consecuencia de la formación de una fase líquida, sugerida por Perera[3], que posiblemente se forma por debajo de la temperatura de sinterización entre la alúmina y la titania junto al aditivo $\left(\mathrm{Na}_{2} \mathrm{O}\right)$. Dentro de los granos de titanato de aluminio se detallan una gran cantidad de partículas de color gris oscuro y blanco, que corresponden a la alúmina y la titania, respectivamente. La fase líquida en referencia está señalada en la fig. 5. Esta se encuentra intergranularmente y se caracteriza por la carencia de una forma definida a diferencia de los granos faceteados de titanato de aluminio. Según Perera[3], la fase líquida podría dar lugar a una sinterización en la cual el líquido debe formar una fase líquida cristalina refractaria que ayudaría a evitar la descomposición del material a la temperatura del tratamiento térmico[3]. Este no es el caso para la composición TAN que después del tratamiento térmico presentó un 5.3\% de fase titanato de aluminio.

Por otro lado, una microestructura de granos bien definidos pero más homogénea que la presentada en la fig. 5 es mostrada en la fig. 6 y corresponde a la composición TAC. Al igual que la composición TAN la presencia de una fase líquida formada entre la alúmina, la titania y el aditivo es responsible de la delineación de los granos de titanato de aluminio.

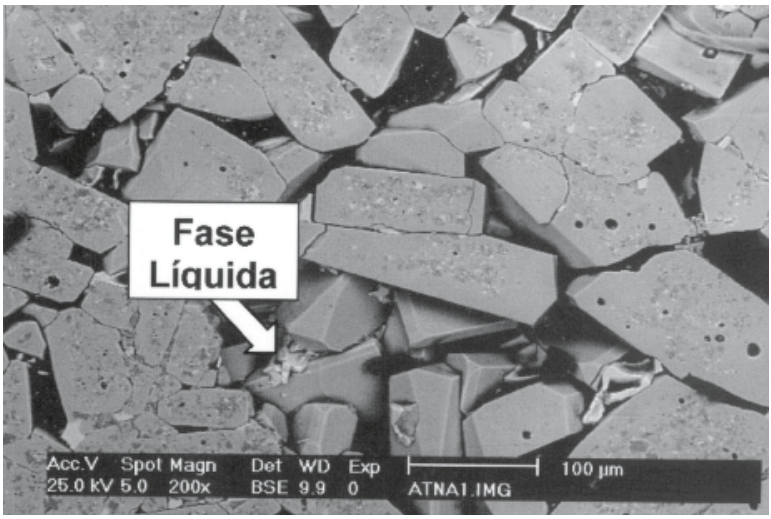

Fig. 5. Microestructura de la composición TAN antes del tratamiento térmico. Nótese la heterogeneidad en los tamaños de granos bien delineados y su diferencia con la fase líquida.

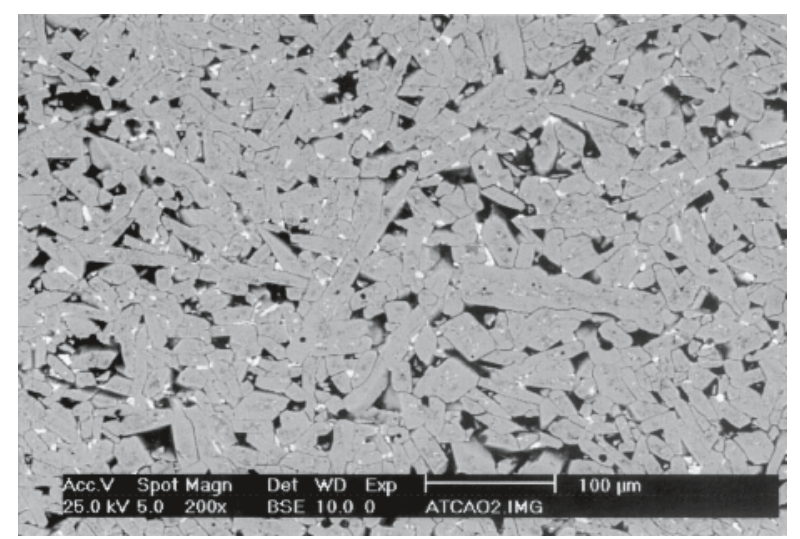

Fig. 6. Microestructura de la composición TAC antes del tratamiento térmico. A diferencia de la fig.5, la microestructura de la composición TAC no presenta crecimiento exagerado de granos.
Perera[3] afirma que la sinterización reactiva en presencia de fase líquida puede producir $\mathrm{Al}_{2} \mathrm{TiO}_{5}$ con una microestructura de grano fino y mínima porosidad. Este es el caso de la composición TAC que presentó el segundo porcentaje más bajo de absorción de agua 2.8\% [14] y una microestructura similar a la descrita por Perera. El $18.9 \%$ de fase titanato de aluminio presente en la composición TAC después del tratamiento térmico se le puede atribuir a la presencia de una fase líquida formada entre los óxidos iniciales y el $\mathrm{CaO}$.

Estos resultados indican que el $\mathrm{V}_{2} \mathrm{O}_{5}, \mathrm{Na}_{2} \mathrm{O}$ y $\mathrm{CaO}$ forman fases líquidas a bajas temperaturas que ayudan a la densificación del material pero ninguno de ellos entra en solución en el titanato. Los radios iónicos del $\mathrm{Ca}^{2+} \mathrm{y} \mathrm{Na}^{+}$son 0.99 y $0.95 \AA$ respectivamente mucho mayores que el del $\mathrm{Al}^{3+}$ lo que imposibilita su ingreso a la red de pseudobrookita, por lo que la temperatura eutectoide en estos sistemas ternarios sigue siendo $1300^{\circ} \mathrm{C}$.

Las figs. 7 - 9 muestran los resultados de las probetas TAF antes y después del TT de $12 \mathrm{~h}$ y un TT posterior de $20 \mathrm{~h}$. En éstas se puede observar una mejor delimitación de los granos de $\mathrm{Al}_{2} \mathrm{TiO}_{5}$ en comparación con la composición original (ver fig.2). La ausencia de oxidos remanentes en la matriz del titanato de aluminio evidencia el efecto catalizador de la ilmenita en la cinética de formación del titanato de aluminio.

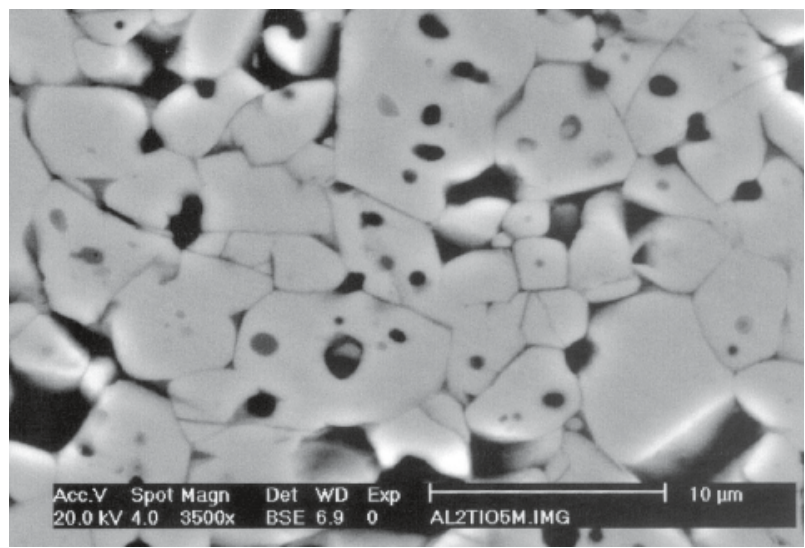

Fig. 7. Microestructura del titanato de aluminio dopado con $3 \%$ de $\mathrm{FeTiO}_{3}$. Nótese los granos delimitados y la ausencia de óxidos iniciales.

Por otro lado, en las fig. 8 y 9, se muestran la microestructura para la composición TAF después de los tratamientos térmicos de 12 y 20 h, respectivamente. La estabilización térmica del titanato de aluminio se ve favorecida enormemente gracias al $3 \%$ de $\mathrm{Fe}_{2} \mathrm{TiO}_{3}$ en la mezcla equimolar de $\mathrm{Al}_{2} \mathrm{O}_{3}$ y $\mathrm{TiO}_{2}$.

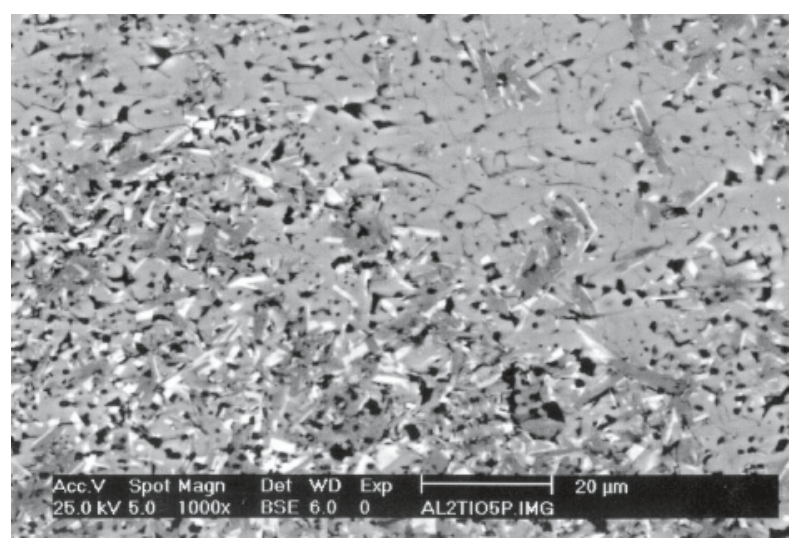

Fig. 8. Microestructura de la composición TAF después del tratamiento térmico de $12 \mathrm{~h}$. Se observan pequeñas zonas de color blanco y gris oscuro indicando una leve descomposición. 
Las cantidades de óxido producto de la descomposición que se observan en la microestructura (ver fig. 8 y 9), son pequeñas aún luego del tratamiento térmico de 12 y $20 \mathrm{~h}$, lo cual se corresponde con los análisis de DRX (ver Tabla IV). En el caso del tratamiento térmico de $20 \mathrm{~h}$, la composición TAF presentó una descomposición del $16.8 \%$, la cual se observa en la fig. 9 como pequeñas regiones aisladas dentro de una matriz de titanato de aluminio. Estas regiones aisladas presentan las mismas características microestructurales descritas para la descomposición de la composición TA tratada térmicamente ( fig. 3).

El posible mecanismo de estabilización se presume sea similar al descrito por varios investigadores $[5,16]$, donde ocurre la formación de una co-solución (isoestrutural) de titanato de aluminio y titanato de hierro que estabiliza térmica y estructuralmente al titanato de aluminio. Paso previo a la formación del titanato de hierro es la descomposición de la ilmenita en $\mathrm{TiO}_{2}$ y $\mathrm{FeO}$. Se presume que el FeO se oxida a $\mathrm{Fe}_{2} \mathrm{O}_{3}$ durante la sinterización en atmósfera oxidante. Luego, a temperaturas entre 900 y $1000^{\circ} \mathrm{C}$, viene la formación del titanato de hierro $\left(\mathrm{Fe}_{2} \mathrm{TiO}_{5}\right)$ que formará posteriormente la solución sólida con el titanato de aluminio $\left(\mathrm{Al}_{2} \mathrm{TiO}_{5}\right)$.

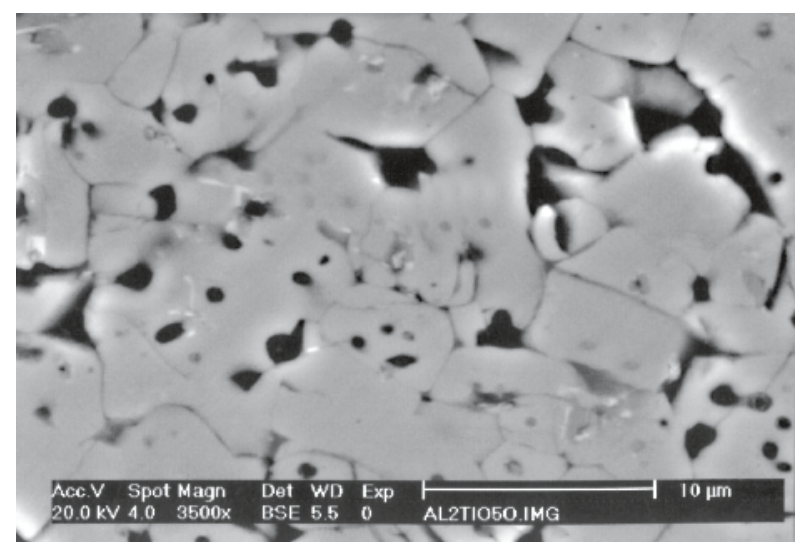

Fig. 9. Microestructura de la composición TAF después de 20 h. de tratamiento térmico.

\section{CONCLUSIONES}

Con la adición de 3\% de óxido de sodio o pentóxido de vanadio no se logró la estabilización térmica del titanato de aluminio. El óxido de calcio estabilizó parcialmente (20\%) la fase titanato de aluminio en los compactos sinterizados tratados térmicamente a $1000^{\circ} \mathrm{C}$ durante 12 horas.

La ilmenita promovió la formación y estabilización total del titanato de aluminio bajo las condiciones experimentales ensayadas en este trabajo. Esta estabilización ocurre porque el hierro entra en solución sólida en lugares isoestructurales a los del aluminio y también rebaja considerablemente la temperatura eutectoide con lo que aumenta significativamente la estabilidad térmica de la solución sólida.

\section{BIBLIOGRAFÍA}

1. E.Kato, K.Daimon, J.Takahashi, "Decomposition Temperature of b- $\mathrm{Al}_{2} \mathrm{TiO}_{5}$ ” J.Am.Ceram.Soc., 63 [5-6], 355-356 (1980).

2. M.Ishituka, T. Sato, T. Endo, M. Shimada, "Synthesis and Thermal Stability of Aluminum Titanate Solid Solutions", J.Am.Ceram.Soc., 70 [2], 69-71 (1987).

3. D. Perera, "Reaction-sintered aluminum titanate", J. Mater. Sci. Lett., 8, 10571059 (1989).

4. H. Wohlfromm, J. Moya, P. Pena, "Effect of $\mathrm{ZrSiO}_{4}$ and $\mathrm{MgO}$ additions on reaction sintering and properties of $\mathrm{Al}_{2} \mathrm{TiO}_{5}$-based materials", J.Mater.Sci., 25, 3753-3764 (1990).

5. G. Tilloca, "Thermal stabilization of aluminum titanate and properties of aluminuim titanate solid solutions", J.Mater. Sci., 26, 2809-2814 (1991).

6. G. Grimes, J. Pilling, "Defect formation in $\mathrm{b}-\mathrm{Al}_{2} \mathrm{TiO}_{5}$ and its influence on structure stability" J.Mater.Sci., 29, 2245-2249 (1994).

7. S. Djambazov, D. Lepkova, I. Invanov, “A study of the stabilization of aluminum titanate" J.Mater.Sci. 29, 2521-2525 (1994).

8. L. Zhien, Z. Qingmin, Y. Jianjun, "The effects of additives on the properties and structure of hot-pressed aluminium titanate ceramics", J.Mater.Sci., 31, 90-94 (1996).

9. V. Buscaglia, M. Alvazzi Delfrate, P. Nanni, M. Leoni, C. Bottino, "The effects of $\mathrm{MgAl}_{2} \mathrm{O}_{4}$ on the formation kinetics of $\mathrm{Al}_{2} \mathrm{TiO}_{5}$ from $\mathrm{Al}_{2} \mathrm{O}_{3}$ and $\mathrm{TiO}_{2}$ fine powders", J.Mater.Sci., 31, 1715-1724 (1996).

10. I. Low, D. Skala, D. Zhou, "Synthesis of functionally gradient aluminium titanate/alumina composites", J.Mater. Sci.Lett., 345-347 (1996).

11. H. Lim Lee, J. Yeol Jeong, H. Min Lee, "Preparation of $\mathrm{Al}_{2} \mathrm{TiO}_{5}$ from alkoxides and the effects of additives on its propierties", J.Mater.Sci., 32, 5687-5695 (1997).

12. M. Andrianainarivelo, R. Corriu, D. Leclercq, P. Mutin, A. Vioux, "Nonhydrolytic Sol-Gel Process: Aluminium Titanate Gels", Chem. Mater., 9, 1098-1102 (1997).

13. I. W. Brown \& D. G.Mc Gavin, Effect of Iron oxide additives on $\mathrm{Al}_{2} \mathrm{TiO}_{5}$ formation. Fourth Euroceramics Ceramics., Vol 4, 487-492, Basic Science, Trends in Emerging Materials and Applications, Edit. by Bellosi., (1995).

14. I. Barrios de Arenas, R. Sicardi, F. Arenas, A. R. Di Giampaolo y S-A. Cho, "Aluminum Titanate Modified by $\mathrm{Na}_{2} \mathrm{O}, \mathrm{CaO}, \mathrm{V}_{2} \mathrm{O}_{5}$ and $\mathrm{FeTiO}_{3}$ "., Ceramics Getting into the 2000`S, Part D, 16, 87-94, Edit. Faenza (1999).

15. P. Pena, H.Wohlfromm, R. Torrecillas, S. Moya , "Microstructure and Mechanical Behavior of Eutectoid Corundum-Rutile Composites", Ceramics International, 26, 375-380 (1990).

16. V.Buscaglia, M. Alvazzi Delfrate, P.Nanni, M. Leoni, C. Bottino, "Factors affecting microstructure evolution during reaction sintering $\mathrm{Al}_{2} \mathrm{TiO}_{5}$ ceramics" Ceramics: Charting the future, 1867-1874, Edit. Faenza (1995).

17. R. J. Brook, "Concise Encyclopedia of Advance Ceramic Materials", Pergamon Press, 20-21, (1988).

18. H.Okamura, E.Barringer, K.Bowen, "Preparation and Sintering of narrowsized $\mathrm{Al}_{2} \mathrm{O}_{3}-\mathrm{TiO}_{2}$ Composite Powders", J.Mater.Sci., 24, 1867-1880 (1989).

19. B.Freudenberg, A. Mocellin, "Aluminium Titanate Formation by Solid-State Reaction of Fine $\mathrm{Al}_{2} \mathrm{O}_{3}$ and $\mathrm{TiO}_{2}$ Powders", J.Am.Ceram.Soc., 70 [1], 33-38 (1987).

20. A.A. Fotiev et al "Phase Diagrams for Ceramists. Figura 6828

21. J.Jung, A.Feltz, B.Freudenberg, "Improved Thermal Stability of Al- Titanate Solid Solutions". Cfi/Ber. DKG 70 N . 6 (1996)

Aceptado: 14.04 .00 